Rev. Est. de Políticas Públicas, 5(1): diciembre 2018 - junio 2019, 7-24

http://dx.doi.org/10.5354/0719-6296.2019.52422

ISSN edición web: 0719-6296

CCopyright 2019: Universidad de Chile, Santiago (Chile)

\title{
Ingeniería de procesos para enfrentar la corrupción y el fraude
}

\author{
Juan Parra \\ Consultor independiente
}

\begin{abstract}
Resumen
El problema que se plantea en este trabajo está relacionado con la corrupción y el fraude, tema contingente en todo el mundo y aparentemente insoluble. Pero así como es un problema, también es una oportunidad para que las sociedades puedan enfrentarla y comenzar a bajar los niveles de corrupción y fraude en sus instituciones. Pocas iniciativas, tanto metodológicas como automatizadas para monitorizar riesgos de corrupción/fraude, existen, por lo que la presente propuesta ofrece un acercamiento desde el análisis, las metodologías y la ingeniería, tanto de procesos de negocios y modelos abstractos, así como de la tecnología como herramienta capaz de analizar información, identificar actos contradictorios y combatir el comportamiento no probo. El estado del arte de la tecnología permite enfrentar el problema, donde el protagonista principal puede ser la ciudadanía y otros agentes interesados en escrutar el comportamiento institucional. De esta forma, el público promedio, tendrá la sensación de que las cosas pueden cambiar y que los agentes cuyo comportamiento ha sido lesivo, puedan ser identificados, sancionados y comenzar a confiar en sus instituciones
\end{abstract}

Palabras clave: Corrupción/fraude, procesos, tecnología

Process engineering to face corruption and fraud

\begin{abstract}
The problem that arises in this work is related to corruption and fraud, a contingent issue throughout the world and apparently insoluble. But just as it is a problem, it is also an opportunity for societies to face it and begin to lower the levels of corruption and fraud in their institutions. Few initiatives, both methodological and automated to monitor corruption / fraud risks, exist, so that this proposal offers an approach from analysis, methodologies and engineering, both business processes and abstract models, as well as technology as a tool capable of analyzing information, identifying contradictory acts and combating unproved behavior. The state of the art of technology allows facing the problem, where the main protagonist can be citizens and other agents interested in scrutinizing institutional behavior. In this way, the average public will have the feeling that things can change and that agents whose behavior has been contradictory, can be identified, sanctioned and begin to trust their institutions
\end{abstract}

Keywords: Corruption/fraud, processes, technology

*Dirección de correspondencia [Correspondence address]:

Juan Parra, Consultor independiente

E-mail: jparral@fen.uchile.cl

Fecha recepción: 29 de septiembre de 2018

Fecha aceptación: 22 de marzo de 2018 


\section{Introducción}

La corrupción y el fraude son unos de los flagelos que entorpece el desarrollo de las naciones en su afán por resolver los problemas de cada sociedad. En todos los continentes se han develado casos de corrupción de mayor o menor calibre y, hasta el día de hoy, no se avizora una retirada del fenómeno, sino que, por el contrario, sigue su paso firme e incólume, afectando la confianza pública y desviando ingentes recursos públicos hacia intereses privados de minorías.

El panorama anterior es justamente lo que constató Peter Eigen, Fundador de Transparencia Internacional, luego de trabajar gestionando recursos desde el Banco Mundial, orientados a financiar proyectos y programas públicos en beneficio de los más necesitados de África. Esto lo motivó a fundar Transparencia Internacional, además de ser una de las primeras personalidades en denunciar públicamente que "...la corrupción es la causa principal de la pobreza, miseria e injusticia del mundo entero" (Navea, 2014: $8)$.

Sin embargo este problema sigue presentándose cada nuevo año en todo el mundo y se puede apreciar en los noticieros donde desfilan políticos, empresarios y ejecutivos en posiciones de poder, los cuales han defraudado la fe pública para beneficio propio. Durante julio 2018, se han visto vulneradas las plataformas informáticas de la banca nacional chilena costándole millones de dólares, además de la fuga de información de miles de clientes, lo que dio como resultado la constatación de la antigüedad y debilidad de las medidas que debieron adoptarse para la protección de los activos involucrados. El importe de recursos defraudados, es parte del botín que el acto no probo imprime a las arcas público-privadas del mundo, las cuales se estiman en más de US\$ 7 billones anualmente (Association of Certified Fraud Examiners, 2018: 4).

También han proliferado programas académicos en universidades, relacionados con la probidad, ética y transparencia, donde se intenta preparar a profesionales con elementos de lucha apropiados y eficaces para enfrentar este problema en el ámbito público y privado. Sin embargo, de dicha oferta programática, casi el $100 \%$ de ella, no comprende dentro de sus contenidos, herramientas de la ingeniería y sistemas automatizados para enfrentar el problema, sino que, más bien, solo abunda teoría para caracterizar el problema e iniciativas administrativas que, en la forma de diseño de controles internos, procedimientos administrativos y ajustes legales, intentan enfrentar el fenómeno.

De modo que, derivado de lo anterior, este documento representa un aporte para los distintos públicos interesados en identificar nuevas fórmulas para combatir la corrupción y el fraude, e ilustrar de qué forma las herramientas de la ingeniería de procesos, en lo específico, pueden servir para este propósito ${ }^{1}$.

\section{Ingeniería de procesos}

Este trabajo aborda, en lo específico, el uso de la disciplina de gestión de procesos aplicada a la resolución de problemas organizacionales, como lo podrían ser, los niveles de corrupción y el fraude en una entidad.

El concepto de proceso es singularmente útil e importante, dado que, en muy resumidas cuentas, es la antesala de los bienes y servicios construidos por una organización. Es decir, cualquier producto o servicio final, consumible por terceros.

De la Figura 1 precedente se puede visualizar que, efectivamente, los bienes y servicios que son consumidos, todo ellos, sin excepción, son construidos por el proceso-i. Si se aplica esta relación a la "construcción de un servicio de corrupción/fraude", se puede analizar que la corrupción, vista esta como un servicio que ha sido construido al interior de una organización, es la resultante de un proceso determinado. Naturalmente, es un hecho desafortunado, no buscado oficialmente, pero existente.

Efectivamente, el acto de la corrupción/fraude, como "servicio", también "debe" construirse al interior de una organización y es la resultante, por lo tanto, de un proceso interno en la organización, donde el acto no probo se expresa en una "transacción" entre el sujeto pasivo (quien recibe la presión y cede) y el sujeto activo (quien ejerce la presión y ofrece estímulo). Se constata, por lo tanto, la "recreación en la acción" que tal contraprestación ilícita refleja en el sentido teleológico implícito en dicha acción social y la correspondencia biunívoca de la misma (Mujica, 2005, p.1).

\footnotetext{
${ }^{1}$ Documento elaborado en base a información, conocimientos y desarrollos metodológicos y tecnológicos generados durante la ejecución del proyecto "Consolidación y aplicación del modelo sobre monitoreo de riesgo de corrupción en gobiernos regionales", presentada por la Universidad Católica de Temuco, al II Concurso de Ciencia Aplicada del Programa IDeA de FONDEF/CONICYT 2013, y aprobada con Código CA13I10108 mediante Resolución Exenta $N^{\circ} 5774 \mathrm{E} / 2013$
} 
Figura 1: Relación proceso-producto

\begin{tabular}{|l|} 
Proceso $_{i}$ \\
\end{tabular} $\begin{gathered}\text { Bienes y/o } \\
\text { Servicios }\end{gathered}$

Fuente: Elaboración propia.

Figura 2: Relación proceso-servicio de corrupción/fraude

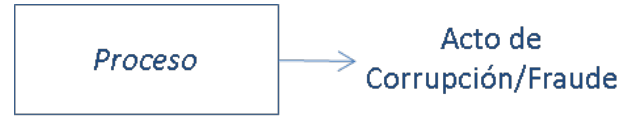

Fuente: Elaboración propia.

Asimismo, también es posible que el acto no probo pertenezca a una cadena de sub procesos y que, en un momento determinado, asociado a un proceso equis, este se concrete, como se ilustra a continuación.

De la ilustración anterior, es posible visualizar que el acto no probo ulterior, puede estar oculto dentro de una cadena de procesos internos y el desafío consistirá en identificar en cuál o cuáles procesos se oculta tal "transacción no proba". Aún más, como se sabe que un proceso posee actividades, será menester identificar qué actividad específica es aquella donde, de facto, se produce la transacción no proba. Lo anterior se ilustra a continuación.

De hecho, es posible que el acto no probo sea una resultante en más de un proceso, según se ilustra a continuación.

De cualquier forma, y dado que una organización es una sumatoria de procesos ${ }^{2}$, será necesario identificar, mediante una metodología determinada, en qué proceso se oculta una actividad específica donde el acto no probo toma lugar.

Sin perjuicio de lo extensa o breve que sea la cadena de procesos y la cantidad de "servicios de corrupción/fraude" construidos, siempre es posible escrutar la "caja negra", es decir, el proceso que es antesala del producto/servicio.

En este esquema se avizora como necesario conocer, ex ante, qué acto no probo es plausible encontrar, como una forma de catalogar un proceso como "proceso crítico", desde el punto de vista de la corrupción/fraude. Lo anterior significa pregun-

\footnotetext{
${ }^{2}$ Por ejemplo, el autor de este documento modeló 163 procesos en el Servicio Agrícola y Ganadero, SAG, en un proyecto bpm, business process management (2012), y 25 procesos sólo en la Unidad de Negocios de "Marcas", en el Instituto Nacional de Propiedad Industrial.
}

tarse, ¿cómo saber qué proceso debe ser objeto de escrutinio?, dado que no todos los procesos pueden ser corruptos. La respuesta es constatar en primer lugar, qué "servicio de corrupción/fraude" ha sido identificado y asociado a qué proceso específico. Lo anterior se ilustra a continuación.

Donde Acto no proboj $=$. Es decir, los actos no probos representan una sumatoria de casuística no proba, que parte con la unidad y que puede terminar con un número significativo (100 ó más), y que es la resultante final del procesoi (el procesoi "construyó" el "acto no proboj").

La ilustración siguiente aporta un listado no exhaustivo de la casuística que es posible documentar y/o levantar en una investigación in situ.

Boniolo aporta una interesante investigación que culmina con un listado y caracterización detallada de prácticas corruptas en Buenos Aires, Argentina (Boniolo, 2010: 379).

\section{Metodología propuesta}

El entendimiento de la naturaleza del acto no probo es, en sí misma, un proceso complejo debido a que estas prácticas son clandestinas, secretas, soterradas, alejadas del prisma de la transparencia, sino que, más bien, están ocultas en las opacidades administrativas de la organización. Pero aun así, es plausible ofrecer acercamiento a una comprensión detallada y cabal del fenómeno que, para este caso, implica "crear" metodología para este propósito.

Se entiende por metodología la "una sucesión de pasos ligados entre sí por un propósito" (Pardinas, $1984 ; 56)$. El uso de esa sucesión de pasos o metodología es una medida de racionalidad. Lo que se quiere decir es que, a diferencia de un sin número de iniciativas plausibles a las cuales se pueda enfrentar 
Figura 3: Relación cadena de procesos-servicio de corrupción/fraude

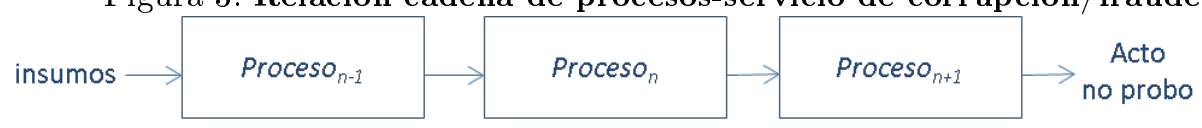

Fuente: Elaboración propia.

Figura 4: Jerarquía de procesos, subprocesos y actividades

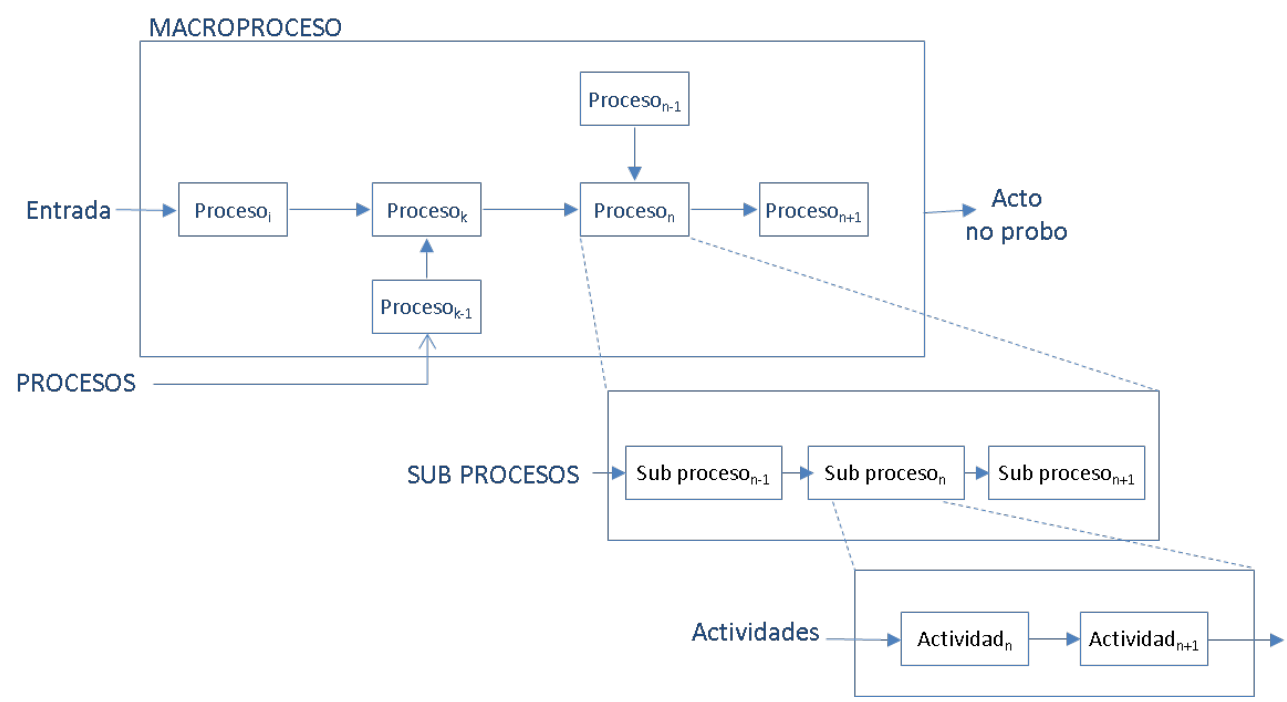

Fuente: Elaboración propia.

Figura 5: Eelación cadena de procesos con más de un acto no probo

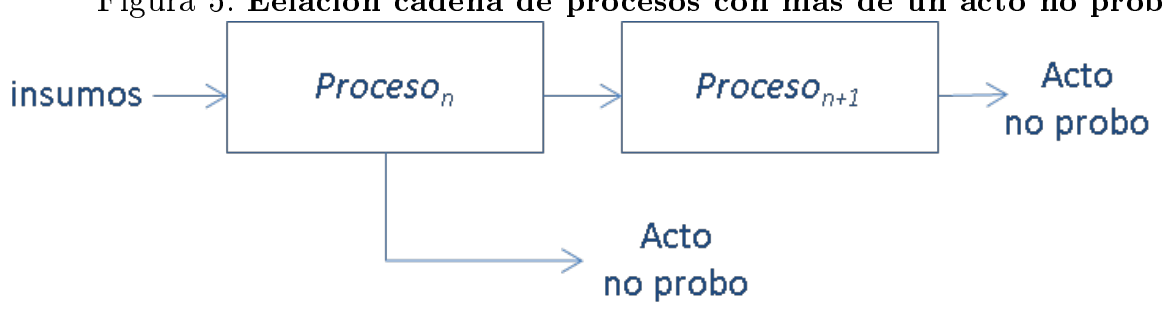

Fuente: Elaboración propia.

Figura 6: Relación procesoi asociado a acto no proboj

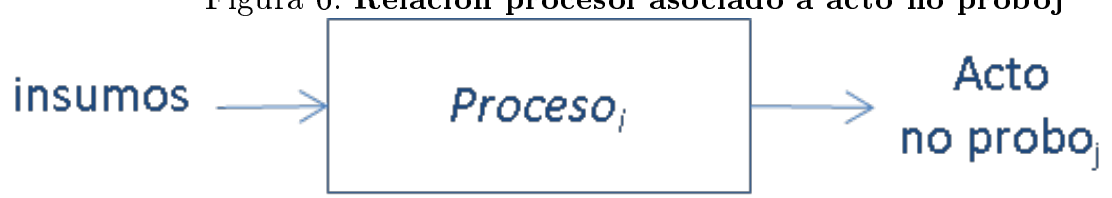

Fuente: Elaboración propia.

Figura 7: Relación procesoi asociado a actos no probosj con ejemplos de casuística

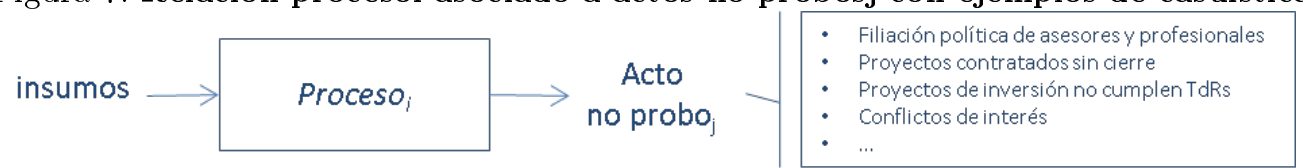

Fuente: Elaboración propia. 
(iniciativas, proyectos, investigaciones que enfrentan profesionales y consultores), la mayoría de las veces es factible identificar y utilizar una metodología de cómo enfrentar ese desafío. Así, si se quisiera, por ejemplo, "medir la satisfacción de usuarios en obras viales como una evaluación ex post de proyectos de inversión pública" (CEPAL y Ministerio de Desarrollo Social de Chile, 2017: 177), se puede utilizar metodologías que han sido diseñadas y utilizadas para este fin; así mismo, si se quisiera analizar los problemas asociados a la toma de decisiones empresariales y su resolución, etc., existen muchas metodologías que se pueden utilizar (Kepner y Tregoe, 1965: 2).

Sin embargo, para evaluar entornos organizacionales, comprendiendo y develando la naturaleza de la corrupción/fraude, no existen metodologías publicadas, con un nivel detalle, guía y precisión, que se puedan usar para este propósito, de modo que, en este caso, se ha debido diseñar una metodología ad hoc que sirva para esta faena y que, en lo sucesivo, y si es útil y prueba ser eficaz, otros investigadores puedan utilizar.

\subsection{Ingeniería Inversa}

Se propone utilizar el concepto de "ingeniería inversa" de Chifofski, aquella citada por Goncalvez Panta (2016) que plantea el "análisis de un sistema para identificar sus componentes actuales y las dependencias que existen entre ellos, para extraer y crear abstracciones de dicho sistema e información de su diseño" (p.1), para abordar la comprensión del acto no probo, su caracterización, su modus operandi, su relación con actores involucrados, identificando roles, oportunidad, y donde, actividades administrativas y técnicas lícitas, son objeto de "presión indebida" por parte de agentes públicos y privados para obtener beneficios derivados de la aprobación administrativa-técnica de iniciativas públicas, para enarbolarlas como propias para la cosecha de votos en una localidad, por ejemplo.

De modo que se propone, en primer lugar, identificar un caso de corrupción/fraude (acto no probo) clásico, descubierto mediante una denuncia, investigación y sanción -por ejemplo. Para lo anterior se pueden revisar los dictámenes de la Contraloría General de la República, $\mathrm{CGR}^{3}$ que sanciona casos concretos de corrupción en el sector público (malversaciones, fraude al fisco, etc.)-, relacionado con un caso que se gestó y desarrolló en un proceso interno de la organización y, a partir de ahí, comenzar

\footnotetext{
${ }^{3}$ https://www.contraloria.cl/portalweb/web/cgr/portada
}

su análisis forense, "hacia atrás", hacia su origen. La ilustración siguiente permite apreciar gráficamente estas relaciones.

De la ilustración precedente se puede visualizar que (análisis de derecha a izquierda):

En la práctica, y asociado a una investigación o análisis formal anticorrupción/fraude, lo que se constatará es la identificación del $100 \%$ de procesos de negocio asociado a un área o temática de interés. Sin embargo, como se aprecia en la ilustración 8, no todos esos procesos ocultan actividades corruptas, es decir, del $100 \%$ de procesos, puede haber un porcentaje de ellos que sí lo son y, en ese evento, serán catalogados como "procesos críticos", desde el punto de vista de la corrupción/fraude. En dicha ilustración 8, sólo uno de tres procesos (el proceso achurado) fue identificado como proceso crítico, no así los otros dos.

Lo anterior es importante porque se requiere precisión para seguir avanzando en el análisis que sea llevado adelante, además de representar un elemento de racionalidad, ya que si los procesos no están relevados formalmente, se deberá impulsar un trabajo de relevamiento que involucra costos y tiempo, de modo que en el caso de la ilustración, se deberá levantar uno de tres procesos, y no los tres.

De la ilustración precedente se puede visualizar que, del $100 \%$ de procesos (siete procesos) de una área de interés, solo tres de ellos (procesos 1, 2 y 7 , achurados en verde), son procesos críticos desde el punto de vista de la corrupción/fraude, y que han ido catalogados así a través de previo análisis (trazabilidad de procesos, entrevistas, investigación formal, etc.). A la hora de focalizar los esfuerzos se conoce que los departamentos organizacionales donde el fraude tiene lugar mayormente en una institución, son: Contabilidad (14\%), Operaciones (14\%), Ventas $(12 \%)$ y alta Dirección Ejecutiva (11\%) (ACFE, 2018, p.37).

Lo que se ha hecho hasta ahora es identificar un proceso de negocios crítico, según el cual operó la corrupción/fraude y que fue posible rastrear en un análisis de ingeniería inversa (trazabilidad). Sin embargo, un proceso de negocios es un conjunto de relaciones, de flujos de información, de transacciones de uno o más roles y áreas, inclusive áreas externas a la propia organización donde, además, se despliegan (o no) accesos a sistemas de información, se liberan reportes, se utilizan controles manuales y/o automatizados, se definen y siguen reglas de negocios y don- 
Figura 8: Análisis Forense de Procesos usando Ingeniería Inversa (tracking de procesos)

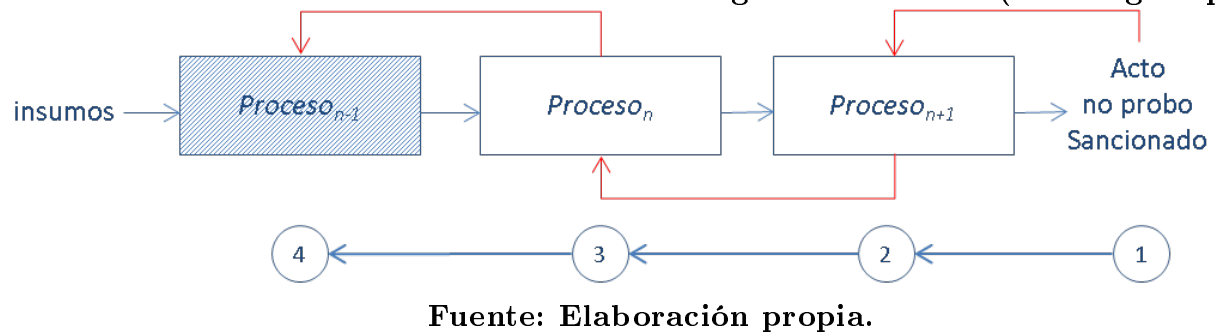

Tabla 1: Explicación Ilustración 8

Numerando Narrativa

\begin{tabular}{|c|c|}
\hline $\begin{array}{l}\text { Acto no probo San- } \\
\text { cionado }\end{array}$ & $\begin{array}{l}\text { Una vez identificado el acto no probo, identificado a través de } \\
\text { un investigación formal de auditoría interna institucional o de la } \\
\text { CGR, o de otra entidad pública (Consejo de Auditoría Interna } \\
\text { General de Gobierno, CAIGG, Unidad de Análisis Financiero, } \\
\text { UAF, otra) -para caso del sector público-, el investigador co- } \\
\text { mienza su análisis con bastante información acerca del acto no } \\
\text { probo, su naturaleza, características, roles involucrados, fecha de } \\
\text { operación, importe, etc., emanadas del informe respectivo. }\end{array}$ \\
\hline $\begin{array}{l}\text { Proceson }+1 \text { - Proce- } \\
\text { son }\end{array}$ & $\begin{array}{l}\text { Del análisis de la información y de las entrevistas con los roles } \\
\text { afectados será posible discriminar y asociar dicho acto no probo } \\
\text { con uno o más procesos de negocio. Este análisis "hacia atrás", } \\
\text { identifica la traza que deja tras sí el acto no probo pudiendo } \\
\text { identificar el proceso relevante donde tuvo lugar el ilícito. }\end{array}$ \\
\hline Proceson-1 & $\begin{array}{l}\text { Finalmente, será posible identificar en forma precisa el proceso en } \\
\text { el cual tuvo lugar la transacción no proba (Proceson-1, achurado), } \\
\text { lo que da origen a su catalogación de proceso crítico, es decir, un } \\
\text { proceso clave, sensible, el cual debería ser objetivo de un ulterior } \\
\text { trabajo de post control vía indicadores de alerta temprana, sis- } \\
\text { temas automatizados de control, diseño de control interno, etc., } \\
\text { para evitar su repetición. }\end{array}$ \\
\hline
\end{tabular}

Fuente: Elaboración propia.

Figura 9: Mapa de Procesos de un área temática de interés, destacando procesos críticos

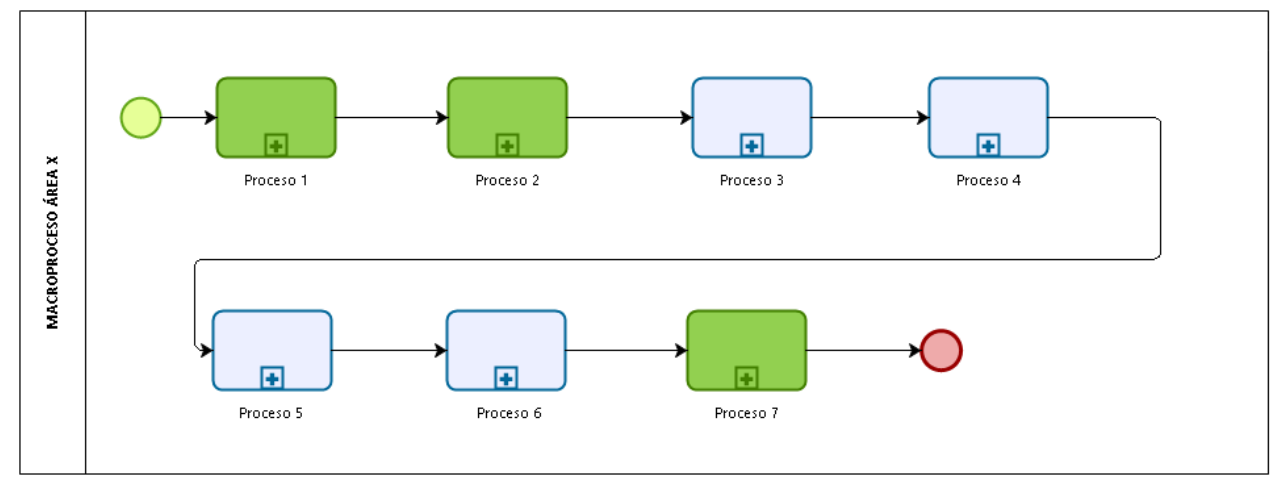

Fuente: Elaboración propia. 
de, esencialmente, se verifican instancias de fricción o relaciones interpersonales concretas entre actores pasivos y activos de corrupción/fraude.

Este nivel de estructuración no es posible verlo con una simple mirada agregada a una caja negra -un proceso a nivel macro-, pero lo que sí es posible desarrollar en consecuencia, es efectuar un relevamiento del citado proceso crítico, de modo de develar todas estas relaciones, llegando a identificar al rol (trabajador/funcionario) específico que fue objeto de presión indebida (o bien, la colaboración de éste en un acto ilícito, con terceros roles) y la actividad concreta específica donde tuvo lugar el acto no probo. Se está hablando, por lo tanto, del modelamiento de un proceso (Beltrán et al., 2002: 19).

Como se puede apreciar, la metodología que se propone tiene un enfoque top down, donde se comienza con un análisis general con base en información cierta, como lo es un caso sancionado de corrupción/fraude, para luego efectuar la trazabilidad "hacia atrás", hasta identificar el dato estructurado inicial -la actividad-, en un proceso bpm modelado.

\subsection{Modelamiento bpm}

Hasta este momento se tiene dos de los tres elementos básicos para identificar a ciencia cierta un acto no probo, bajo esta propuesta metodológica, a saber:

1. Se tiene la casuística no proba (el acto de corrupción/fraude, identificado a través de una investigación formal)

2. Se tiene el proceso de negocio crítico, aquél proceso que oculta un acto de corrupción/fraude, que a su vez pertenece a un macro proceso

3. Pero no se tiene la actividad crítica específica, es decir, aquella tarea desarrollada por un trabajador/funcionario que es susceptible de cometer corrupción/fraude, o bien es objeto de una presión indebida con la finalidad de corromper/defraudar, y que será menester identificar y caracterizar

Estas relaciones causales "hacia atrás", se ilustran a continuación.

Sin embargo, este esquema no permite visualizar el detalle de flujos de información que acaecen dentro del proceso crítico (Procesok), los roles intervinientes y otros detalles administrativos y automati- zados, de modo que será menester diagramarlo como un proceso bpm.

Utilizando herramientas de modelamiento bpm tipo Bizagi, Aris, Camunda y otros, para efectuar el levantamiento de un proceso de negocios, es posible detallar muy precisamente lo que acontece dentro de un proceso de negocios determinado, tal como se ilustra precedentemente. Efectivamente, el diagrama muestra un proceso crítico (Proceso $\mathrm{X}$ ), en el cual participan cinco roles (cargos empresariales/institucionales) que desarrollan una o más actividades (recuadros celestes), donde se verifican tareas, toma de decisiones, disyunciones, controles, flujos de información, etc., y que, en lo específico, una de esas actividades (actividad 9, destacada en rojo) -y que es desarrollada por el rol 5-, representa una actividad donde toma lugar un acto de corrupción/fraude por parte de agentes internos o externos al área, y que representa la casuística de corrupción/fraude que se ha tipificado a través de investigaciones.

Se destaca que el análisis puede llegar a este nivel de detalle y certeza relativa, porque el investigador ha podido "rastrear" la casuística no proba -mediante ingeniería inversa-, ha podido contar con la colaboración de las autoridades internas de la organización (Dirección Convencida) y del propio agente (rol, funcionario, trabajador) afectado, quien ilustra y caracteriza el acto no probo propiamente tal, que le ha tocado experimentar.

Con relación a la naturaleza y características de la actividad crítica, se puede decir que estará asociada a tareas específicas que desarrolla un rol (funcionario/trabajador) dentro de un proceso y que es interesante para el corruptor, porque derivado del resultado de esta tarea, puede detectar debilidades, oportunidades -oler sangre, en definitiva-, para acceder a ulteriores beneficios que puede capitalizar. De modo que el agente corruptor despliega recursos para minar la resistencia del rol o motivar su colaboración, exigiendo que dicho rol adopte medidas contradictorias corruptas/fraudulentas materializándose, de esta forma, el acto de corrupción/fraude.

\subsection{Acerca de las definiciones de corrupción}

Se ha hablado de corrupción/fraude, pero no hemos ampliado el análisis a sus causas más fundamentales, al nivel de las definiciones, debido al enfoque técnico de este documento que propone una solución ingenieril al problema de la corrupción. Sin embargo, la abundante literatura respecto de lo primero, 
Figura 10: Relación causal Acto no probo (casuística)-Proceso Crítico-Actividad Crítica

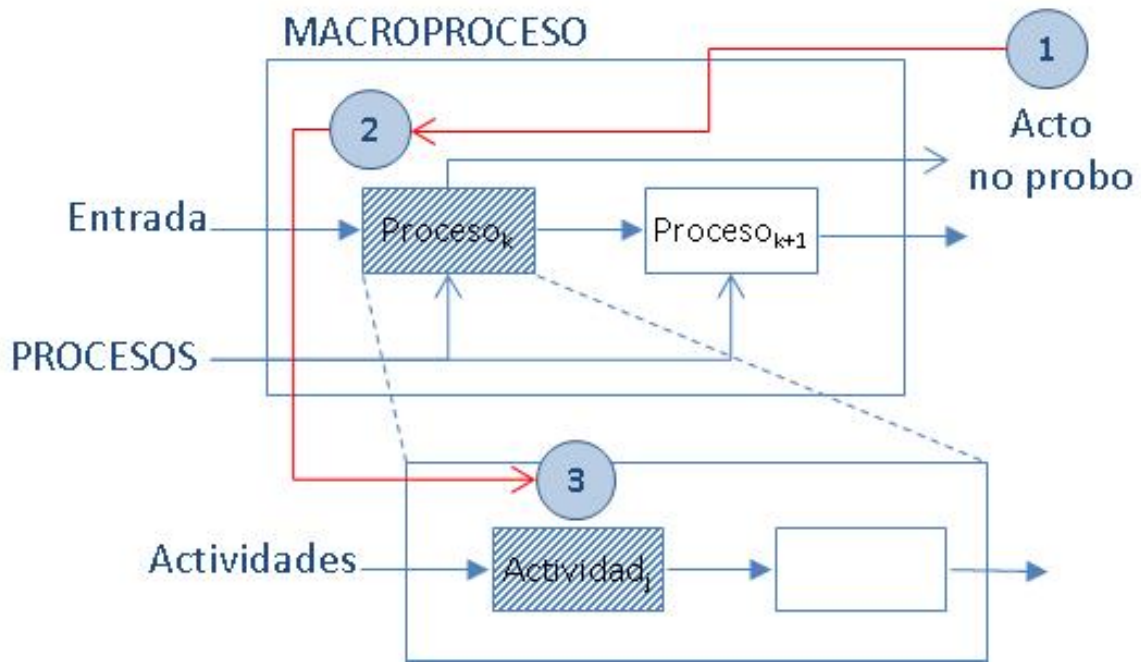

Fuente: Elaboración propia.

Figura 11: Diagramación de un proceso de negocios bpm, con identificación de una actividad crítica

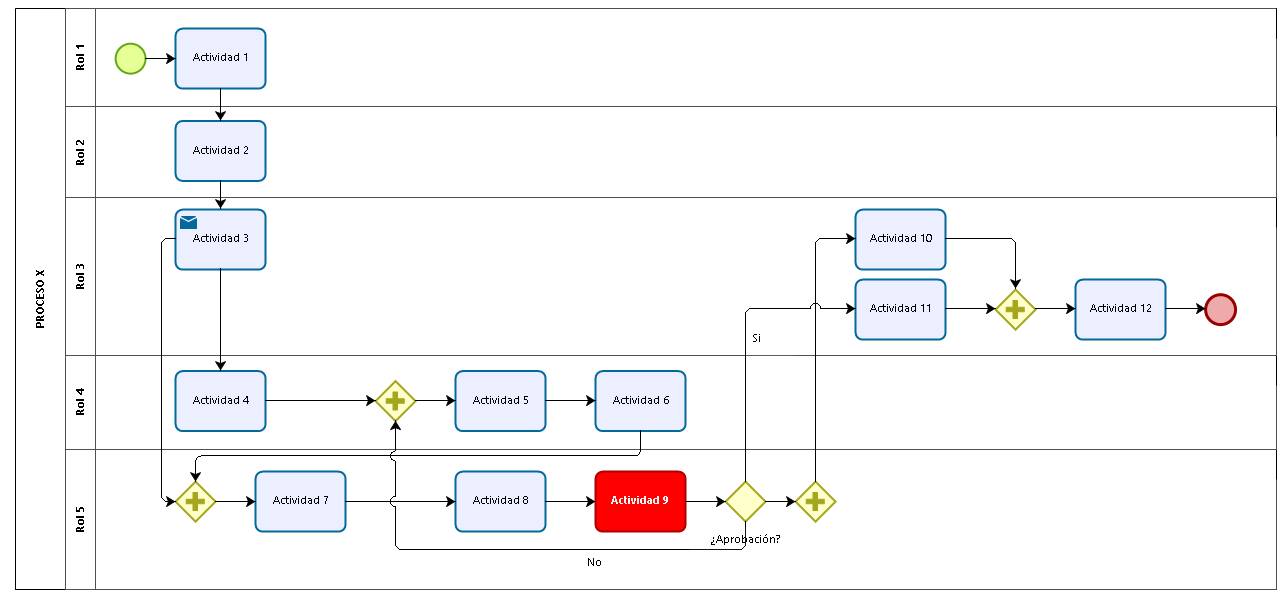

Fuente: Elaboración propia. 
nos puede aportar definiciones que, ponderadas por su propio análisis, pueden servir para mejor ilustrar el nexo simbiótico existente entre técnica y verbo.

Existen definiciones de corrupción existen de larga data y variadas, comenzando con la que ofrece Transparencia Internacional (2009), señalando que es: "abuso del poder para beneficio propio" (p.14). Lo que sabemos es que la corrupción adquiere muchas formas y que, aunque la propia naturaleza la señalare como algo pernicioso que debería no existir e intentar extinguir, también ha sido sindicada como una práctica factible y acaso necesaria, allí, donde el Estado falla en saciar necesidades sociales básicas del ciudadano, tal como Akhil Gupta lo documenta en cita de Mujica (Mujica, 2005: 6). Lo propio se ve refrendado en la sanción social vía votos en Costa Rica quienes, encuesta de opinión de por medio, previo a los comicios, un $23.2 \%$ señala que prefiere un candidato "deshonesto, pero competente" (Martínez Rosón, 2016: 80), mismos votantes que ven en estos prospectos, una promesa de mejor futuro.

El intento por definirla, acaso consensualmente, ha sido arduo, por la complejidad intrínseca del fenómeno la cual ha ido mutando en el tiempo conforme cambian las reglas del juego, el estado del arte y el estado de la tecnología. En este intento ha habido autores que incluso han utilizado la teoría del color atribuyéndole a la corrupción los valores de negra, gris y blanca, de Heidenheimer et al. (1989), citada por Villoría (Villoria, 2000: 26), en torno a una condena transversal de la corrupción de todas las clases sociales, una visión ambigua de la misma, hasta una nula oposición a ésta por parte de la sociedad (corrupción negra, gris y blanca, respectivamente). Ante este panorama, señalo que la definición de corrupción como:

Toda acción u omisión de funcionario público en que este se desvíe de los deberes normales de su cargo con el objeto de obtener gratificaciones de índole privado (familia, amistad) o beneficios políticos, pecuniarios o de status, así como cualquier utilización en beneficio personal o político de información, influencias $\mathrm{u}$ oportunidades surgidas en razón de su cargo.

(Orrego, 2000, p.3)

En esta definición se aprecia el carácter multifactorial del fenómeno el que alcanza factores sociopolíticos, institucionales, jurídicos, económicos y éticoculturales.
Los modus operandi que adopta la corrupción serán aquellos que afecten la "preocupación sociológica clásica: toda aquella práctica que altere el orden social de reglas claras y posibilidad de bienestar social" (Boniolo, 2010: 375) y que pueden materializarse en una transacción evidente y directa, como también ocultarse y disfrazarse en los vericuetos de la ambigüedad como lo señala Mujica al ilustrar una de las tantas acrobacias utilizadas, cuando señala que el sujeto activo ofrece "agilizar" un trámite al sujeto pasivo, hasta el uso de códigos más o menos universales que intentan usar sinónimos generales y ambiguos, y transitar, en una misma transacción, utilizando epítetos para perfeccionar materialmente el negocio (Mujica, 2004: 41), en lo que podríamos aseverar que la corrupción se perfecciona comercialmente con el soborno.

Pero nunca hay que perder de vista de que la corrupción es un acto de defraudación que acaece en "un sistema social sin valores que impide el desarrollo económico de las empresas" (Mora et al., 2017: 37-45) y que son perpetrados por individuos con pobre fortaleza axiológica, "una forma extrema de ausencia de ética" (Villoria, 2000: 27-97), y que ajustan su corbata en el cuello blanco cada nueva mañana, con "sobornos, fraudes, estafas, malversación o desviación de fondos, tráfico de influencias, incumplimientos y otras corruptelas" (Mora et al, p.41), todo lo cual debe ponderarse por un estado del arte de corrupción de ese momento histórico de cada país para "la violación de las normas formales (leyes) o informales (usos y costumbres sociales)" (Boniolo, 2010: 371-372).

Finalmente, y como una forma de diferenciar o esquematizar el acto no probo en su generalidad, me inclino a señalar que la corrupción representa un concepto asimilado a un superconjunto -en lenguaje de lógica booleana-, donde el superconjunto es la corrupción que cobija en su seno todo el conjunto de fórmulas utilizadas por el hechor para lesionar la probidad. En estos términos todas las tipificaciones de corrupción serán elementos de ese universo, ya que intentar buscar diferencias entre un concepto y otro tiene fútil importancia, a mi juicio, y más ayuda una rápida lectura de la caracterización de cada acto no probo existente y pasar, rápidamente, al espacio del diseño de estrategias, tácticas, proyectos y programas, para seguir resistiendo, desde la trinchera del trabajo inteligente y pragmático, a la nueva mutación que la masa informe de la corrupción desarrolla cada nuevo segundo.

En la Tabla 2 se proporcionan actividades críti- 
cas que han sido objeto de presión indebida para cometer corrupción/fraude en el sector público.

El riesgo implícito es que se efectúen pagos por nivel de avance físico de obras que presentan, de hecho, un nivel de avance menor.

En la Tabla 3 se proporcionan actividades críticas que han sido objeto de comisión de fraude (o fraude potencial) en el sector privado.

Lo siguiente por hacer es diseñar mecanismos con los cuales se pueda monitorizar que estas actividades críticas (asociadas a procesos críticos) estén controladas y no presenten nueva casuística asociadas a ellas. Esto se logra diseñando métricas de procesos asociadas a las actividades específicas identificadas, en la forma de indicadores de desempeño o KPIs (Key Performance Indicator).

\subsection{Diseño de Indicadores de Alerta Tem- prana}

En este punto se ha identificado actividades críticas las cuales son desarrolladas por un rol dentro de un proceso, en un área determinada, y que ha sido objeto de presión indebida que culmina con un acto de corrupción/fraude, por lo que, en lo sucesivo será necesario diseñar medidas para evitar su repetición en el futuro, así como la construcción de indicadores de alerta temprana (KPIs) que midan el acto no probo, si este acaece.

El diseño de indicadores de desempeño está asociado al control de gestión que las instituciones públicas y privadas deben ejercer para mantener el control de sus operaciones en el cumplimiento de su misión y visión corporativo. Los indicadores de gestión directivos tienen la virtud de asociar objetivos con desempeño institucional. Tal característica es sumamente interesante para los directivos, ya que pueden ir adoptando los ajustes que los sistemas de control de gestión le señalan cuando hay desviaciones entre las iniciativas de gestión típicas (planeación, programas y presupuestos, con sus objetivos subyacentes) y la información que los ratios arrojan.

De cualquier forma, las enseñanzas que la literatura especializada recomienda (Beltrán, 2002, p.33), se puede utilizar para el diseño de indicadores de alerta temprana de corrupción/fraude, y que se pueden complementar con las instrucciones y recomendaciones ofrecidas por la Dirección de Presupuestos (Dipres) del Ministerio de Hacienda (Ministerio de Hacienda, 2009, p.27), para el caso del diseño de in- dicadores en instituciones públicas.

Se propone una cédula, formato o formulario relacionado con el diseño de indicadores de alerta temprana, el cual representa un elemento de reporte, en este caso, de cómo se caracterizarán los KPIs, es decir, qué información debe ser proporcionada -y controlada- por estos. En otras palabras, es un formulario que contiene una serie de variables de control que serán útiles para administrar el fenómeno de la corrupción/fraude cuando se lo monitorice.

Se ilustra a continuación la cédula de control de KPIs.

Con relación al diseño de los indicadores de alerta temprana, hay un par de consideraciones por tener en cuenta. En primer lugar, se deben considerar las recomendaciones acerca de los métodos más eficaces que han probado ser útiles, en distintas partes del mundo, en torno al combate de la corrupción/fraude, a saber (PriceWaterhouseCoopers, 2010): las denuncias internas (organizaciones gubernamentales/todas las industrias: $31 \%$ y 16 , respectivamente), externas informales ( $14 \%$ y $11 \%$, respectivamente) y los procedimientos formales whistleblowing ( $5 \%$ y $7 \%$, respectivamente). Una publicación de KPMG señala que el $24 \%$ de los casos de fraude son identificados por quejas y hotlines, y el $20 \%$ de ellos debido a whistle-blowing formales (KPMG International, 2016: 16). Con este dato crucial se hace recomendable, por lo tanto, considerar el diseño de indicadores de alerta temprana donde puedan participar funcionarios/trabajadores y público (ciudadanos) en general, los cuales han demostrado motivación para efectuar denuncias.

Se ilustra a continuación, el uso de esta cédula para la actividad crítica 2, "Validar Pago", de la tabla, 2 "Análisis Proceso/Actividad Crítica para el Sector Público", precedente, donde se diseñó un KPI con participación de la ciudadanía.

\subsection{Ranking de casuística no proba para el diseño de KPIs}

Como se señaló precedentemente, asociado a cada proceso crítico es posible identificar variada casuística (modus operandi) de actividad no proba, en cantidad y naturaleza, todas las cuales son susceptibles de medir a través del diseño de KPIs (ilustraciones 6 y 7). En dichas ilustraciones se apuntó que la casuística podrían ser centenares, por lo que teóricamente se podrían diseñar centenares de KPIs. Sin embargo, diseñar una plataforma de control con 
Tabla 2: Análisis Proceso/Actividad Crítica para el Sector Público

Proceso crítico sector público

\begin{tabular}{ll}
\hline Proceso de & 1. Actividad Crítica: Revisión de admisibilidad \\
Admisibilidad de & Actividad desarrollada por el rol "Analista" y consiste en que \\
Proyectos & éste procede a revisar si el proyecto cumple las exigencias de \\
& admisibilidad administrativa y técnica para que sea visado como \\
& proyecto hábil para ser financiado \\
& El riesgo implícito aquí es que determinados proyectos que no \\
& cumplen con el proceso de admisibilidad pasen dicho filtro y sean \\
& financiados por el erario público \\
\hline Proceso Gestión de & 2. Actividad Crítica: Validar Pago \\
Pagos & Actividad desarrollada por el rol "Analista de Inversiones" donde \\
& éste aplica su procedimiento que culmina con la aprobación o no, \\
& del pago que viene en el Estado de Pago \\
& El riesgo implícito es que se efectúen pagos por nivel de avance \\
& físico de obras que presentan, de hecho, un nivel de avance menor \\
\hline
\end{tabular}

Fuente: Elaboración propia.

Tabla 3: Análisis Proceso/Actividad Crítica para el Sector Privado

Proceso crítico sector privado

Proceso de Adquisiciones

\section{Detalle actividad crítica}

1. Actividad Crítica: Aprobar/Rechazar Cotización

Actividad desarrollada por el rol "Unidad Requirente" y consiste en que éste procede a revisar y aprobar o rechazar una cotización para la ulterior compra de bienes y servicios

El riesgo implícito es que aprueben cotizaciones señaladas como compras de "emergencia" que vulneren controles y se defrauden recursos empresariales

Proceso Chequeos Ambientales de Empleados
2. Actividad Crítica: Diseño y aplicación de Chequeo Ambiental Actividad desarrollada por el rol "Analista de Riesgos Internos" quien procede a diseñar y aplicar el procedimiento de chequeo ambiental de empleados de una institución

El riesgo implícito es que no se aplique el procedimiento de chequeos ambientales de empleados lo que puede provocar fraudes internos

Fuente: Elaboración propia. 
Figura 12: Cédula de Control de KPIs

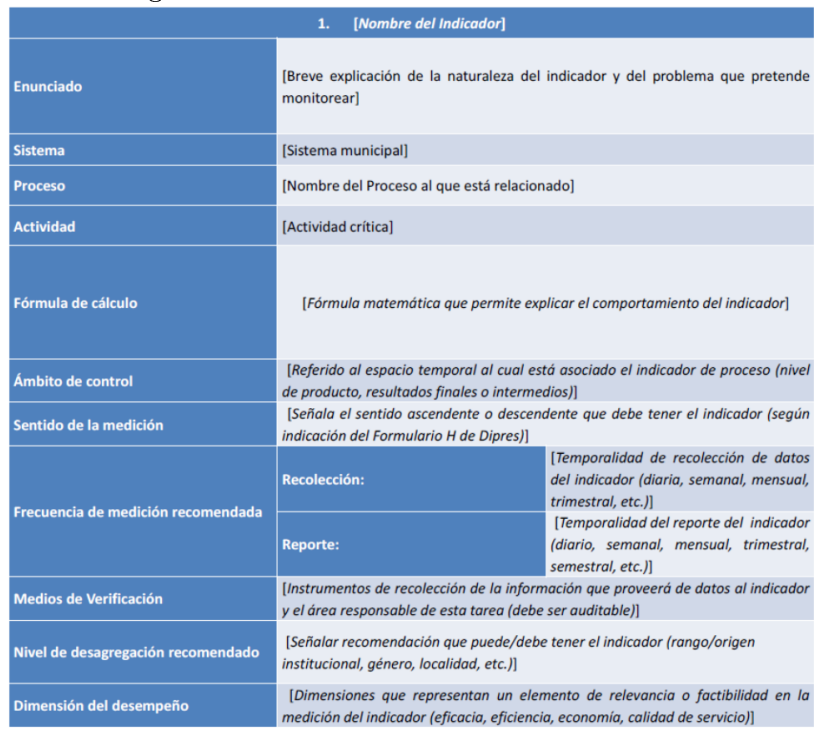

Fuente: Elaboración propia en base a recomendaciones de Dipres.

Figura 13: Ilustración Cédula de Control de KPI de Actividad Crítica para el Sector Público

\begin{tabular}{|l|l|}
\hline \multicolumn{2}{|c|}{ 1. Denuncias ciudadanas respecto de nivel de avance físico publicitado por Entidad } \\
Pública
\end{tabular}

Fuente: Elaboración propia.

Rev. Est. de Políticas Públicas, 5(1): diciembre 2018 - junio 2019 
centenares de KPIs, por ejemplo, para uso directivo en un "Sistema Automatizado de Monitorización de la Corrupción/Fraude", implicaría que el directivo deba enfrentarse a esta abrumadora cantidad de KPIs (digamos, $100 \mathrm{KPIs}$ ) lo cual contraviene las recomendaciones metodológicas de diseño de sistemas de información directivos, por lo que se hace necesario construir un ranking de casuística para acotar a un número breve, pero relevante, de casuística y KPIs concomitantes, que sí sea posible de ser explotado por la administración superior. Naturalmente, para el caso de un sistema para uso táctico u operacional, sí es posible (y recomendable) considerar el $100 \%$ de la casuística y un número de KPIs asociado.

Por lo tanto, si la investigación arrojare centenares de casuística de corrupción/fraude para las cuales se deba diseñar KPIs para uso directivo, será menester aplicar un complemento metodológico para confeccionar un ranking de casuística no proba, que culmine con un set de pocos pero relevantes casos de corrupción/fraude.

De la ilustración precedente se puede visualizar las variables intervinientes para construir un ranking de casos de no probidad, de modo de obtener un set acotado de casos de no probidad caracterizados por criticidad de impacto en corrupción/fraude, para luego diseñar un conjunto de KPIs, asociados a actividades específicas, para una ulterior monitorización a través de, por ejemplo, un sistema automatizado de alerta temprana.

Se procede a continuación a explicar la operatoria del procedimiento para la construcción de un ranking de casuística de no probidad.

\section{Sistemas automatizados de moni- torización de la corrupción/fraude}

Los anteriores apartados han permitido diseñar un modelo funcional de cómo opera la corrupción/fraude en una organización típica, y lo siguiente por hacer es evaluar la posibilidad de transitar desde este modelo abstracto en el papel, hacia un modelo automatizado en la forma de un Sistema de Información Automatizado de Monitorización de la Corrupción/Fraude.

Si se somete el citado modelo a un testeo, por parte de las áreas -que han sido aquellos usuarios entrevistados que entregan la información que caracteriza el acto de no probidad-, y esta prueba su eficacia, entonces lo siguiente es automatizar el modelo para que se puedan capitalizar los beneficios del mismo, a través de una aplicación web y aplicación móvil, debido esto último, a que los usuarios de este servicio, para la denuncia temprana, poseen dispositivos móviles inteligentes (teléfonos inteligentes, tablets, laptops, netbook), y la cantidad de dispositivos va al alza, factor que permitirá multiplicar el uso potencial de esta herramienta de denuncia ciudadana en torno al actuar no probo de los agentes púbico/privados y que, sumado a un procedimiento interno de administración del caso de corrupción denunciado, con trazabilidad del evento, valide las denuncias. De esta forma se puede obtener un mecanismo eficaz para combatir la corrupción/fraude en nuestras instituciones públicas y privadas.

Este aspecto tiene gran importancia dado que los reportes de entidades que estudian el fenómeno de la corrupción/fraude en forma sistemática (Libertad y Desarrollo, en Chile, PriceWaterhouseCoopers Chile e Internacional, KPMG internacional) señalan que, cada vez más, los ataques a la probidad son cometidos usando la tecnología, pero también arrojan evidencia de su poco uso para combatirla. De hecho, de la literatura revisada para este punto específico, se puede decir que hubo publicaciones que no mencionaron en absoluto el uso de tecnología para detectar fraude (PriceWaterhouseCoopers, 2010), pasando desde $3 \%$ (KPMG International, 2016: 21), hasta $46 \%$ usando varios métodos basados en tecnología, como teléfonos hotline, email, formularios on line, redes sociales (Association of Certified Fraud Examiners, 2018: 19).

Los pasos que siguen al levantamiento de los procesos críticos señalados precedentemente, suponen su mejoramiento continuo en el tiempo y su automatización bajo una plataforma workflow, donde la monitorización de un sistema automatizado se pueda ejercer en tiempo real, inclusive.

De la misma forma, se sabe que en una organización típica se generan millones de datos a través de sus distintos sistemas (correos electrónicos, redes sociales internas/externas como twitter, WhatsApp, Facebook, canales youtube, blogs, plataformas interactivas, etc.), todos los cuales, ejerciendo los resguardos que exigen las leyes de protección de datos personales (Ley $\mathrm{N}^{\circ} 19.628$ sobre protección de la vida privada, para el caso chileno), son plausibles de ser utilizadas para la pesquisa de transacciones no probas. Por lo tanto, el citado sistema automatizado anticorrupción/fraude, podrá complementarse con herramientas business intelligence, big data apli- 
Tabla 4: Procedimiento para la Construcción de un Ranking de Casuística No Proba

\section{Numerando procedimiento}

1

2

\section{Explicación}

Listar casos de casuística de no probidad (corrupción/fraude), posible de recabar mediante investigación ad hoc en la forma de sanciones de CGR, UAF, CAIGG, Auditoría Interna, Investigaciones Público/Privadas, que culminen con un listado extenso $(100+$ casos $)$

Asociar cada uno de los casos, con el área organizacional donde éste acaece, y aún más, con el proceso específico donde éste toma lugar. Así, se puede ver, por ejemplo, de la ilustración precedente, que la "Casuística 1", está presente en el "Área 1", y en el "Proceso 1", pero también está presente en el "Área n", en el "Proceso n", etc.

Estas asociaciones las realiza el usuario entrevistado (o equipo de trabajo entrevistado), quienes conocen la dinámica interna, su realidad, dominan sus áreas, procesos, procedimientos y controles (o la ausencia o debilidad de éstos), y conocen las prácticas y clima de corrupción/fraude que se manifiestan en sus entornos laborales

Luego se diseña una Escala de Impacto (representa el nivel de exposición del proceso (área), ante el riesgo de ocurrencia de la casuística de no probidad) que sanciona con un número, el acto de no probidad, desde el guarismo 1 (Sin Impacto), hasta el 5 (Máximo Impacto). Lo anterior significa que se debe confrontar cada caso, contra esta escala y asociar el caso con un número, que representa el nivel de impacto de corrupción/fraude para la organización

De esta forma, se le pregunta al usuario entrevistado (o al equipo entrevistado) ¿qué nivel de impacto de corrupción tiene ese caso de no probidad analizado? Para el caso ilustrado, por ejemplo, las Casuísticas 1, 2 y 3, a juicio del usuario/equipo entrevistado, tiene máximo impacto (guarismo 5), por lo que califica para ser monitorizado a través de KPIs asociados a las actividades críticas de esas áreas y procesos críticos

La decisión ulterior por adoptar, es la elección del corte numeral $(5,4$, etc.) con lo que se obtiene el set de casos no probos críticos y sus áreas y procesos asociados, los cuales serán objeto de modelamiento para identificar las actividades críticas en torno a las cuales se materializan los casos de no probidad, para las cuales de diseñarán los KPIs

\section{Fuente: Elaboración propia.}




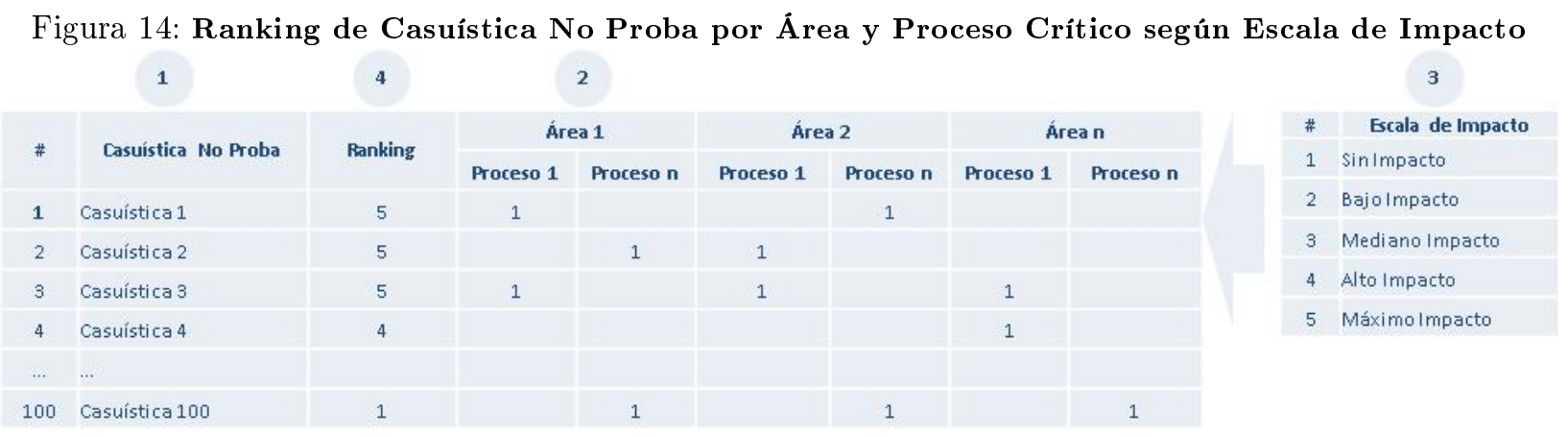

Fuente: Elaboración propia.

cada, minería de datos, redes neuronales, inteligencia artificial, geographic information systems, entre otros.

Lo anterior significa que, dado que el principal insumo para la monitorización de la corrupción/fraude lo representa el flujo clandestino de información -en su gran mayoría-, esta materia prima es la que abunda en los entornos organizacionales, por lo que solo resta el diseño, desarrollo, implementación y explotación de un sistema de información automatizado que utilice esta información con los fines señalados. Adicionalmente, si esta información es puesta a disposición del público, en un ambiente de transparencia de información y de gobierno abierto (con datos abiertos), dicha información permitirá que públicos exógenos a la organización (think tanks, ONGs, centros universitarios de investigación, plataformas $u$ observatorios de vigilancia ciudadana) puedan utilizarla para fines propios y desarrollar cruces de información y su análisis y recomendación concomitantes, que permitan perfeccionar el comportamiento probo de instituciones públicas y privadas.

De modo que, con todas estas herramientas disponibles para sofisticar la lucha contra la corrupción/fraude, es plausible contar con una plataforma automatizada que entregue opciones a agentes internos y externos a la organización, para que éstos escruten si los recursos público/privados se utilizan eficiente y eficazmente y persiguen los fines públi$\mathrm{co} /$ privados para los cuales se asignaron. Esta información se puede desplegar para que dichos públicos confronten la realidad que les toca vivir -de un programa/proyecto público/privado que los beneficia, por ejemplo-, contra la evolución de ese programa y ejercer medidas de control, denunciando el comportamiento no probo nominal (implica que se debe impulsar una investigación interna mediante proce- dimiento ad hoc, para validar/desechar la denuncia), utilizando la plataforma automatizada que se pone a su disposición, para efectuar alertas tempranas de comportamiento contradictorio.

\section{Conclusiones}

Los actos no probos surgen de la opacidad pública/privada en sus procesos, procedimientos, prácticas administrativas, toma de decisiones y febles controles, todos los cuales representan pasto fértil para el actuar contradictorio de agentes públicos y privados que capitalizan estos yerros institucionales afectando su reputación, es decir, "el activo más importante de una institución pública: la transparencia y probidad", como reza en la Guía de Recomendaciones para el Sector Público en la implementación de un sistema preventivo contra los delitos funcionarios, el lavado de activos y el financiamiento del terrorismo ${ }^{4}$. Lo anterior será combatido con el valor negativo de su actual naturaleza, esto es, transparencia y escrutinio público/privado de procesos, procedimientos, mejores prácticas, toma de decisiones y diseño de controles más eficientes y dirigidos, pero, sobre todo, con la participación de agentes públicos (funcionarios) y agentes privados empoderados (ONGs, ciudadanos, trabajadores).

La corrupción y el fraude organizado es un ente que tiene vida propia; es un proceso social que tiene implicancias económicas, sociales y morales que el pudor social obliga a prevenirlas. Sin embargo, conforme aparecen iniciativas que intentan mitigarla, centros de poder opaco comienzan a idear fórmulas de reacción para recuperar el control disoluto de los procesos decisorios relacionados con el uso de recur-

\footnotetext{
${ }^{4}$ https://www.uaf.cl/intituciones publicas/oficio circular n20 guia recomendaciones.aspx
} 
sos fiscales y privados, de modo que será menester seguir sofisticando un modelo de combate, como lo puede ser un sistema automatizado para combatir eficazmente el flagelo.

Sin embargo, aun cuando las relaciones de corrupción/fraude se ocultan en los vericuetos opacos de la organización, sí es posible utilizar elementos de análisis y herramientas tecnológicas de uso cuotidiano, hoy por hoy, como lo es la ingeniería de procesos, la teoría de sistemas, la automatización y la ingeniería de software, para combatirlos eficazmente.

Por otro lado distintas instituciones públicas y empresas privadas han desarrollado iniciativas para mermar esta práctica disoluta y recuperar el control de sus recursos. La auditoría forense se ha erguido como una práctica nueva y concreta para enfrentar el problema y sancionarlo, así como como los canales internos y externos de denuncias de las empresas, los sistemas hotlines, las denuncias (whistle blowing), así como las páginas de denuncia en distintas instituciones (CGR, Consejo para la Transparencia). En este último caso, las plataformas de acceso para denunciar no permiten la protección del denunciante sino por el contrario, dichas plataformas exigen, para completar la denuncia, toda la información del denunciante, aspecto que puede hacer desistir de la denuncia habida consideración de la nula protección de confidencialidad del denunciante, ante presiones o amenazas plausibles. De modo que, si la administración superior impulsa un enfoque que "procure crear una cultura de integridad y franqueza que motive a todos los empleados para «hacer lo correcto»" (PriceWaterhouseCoopers, 2010, p.5), es probable que no surta el efecto deseado, dada la indefensión del denunciante una vez declarado el caso; se requieren garantías para proteger, en todos los aspectos, al colaborador motivado.

Por ejemplo, la plataforma Ciudadanía Inteligente sí posee un servicio que promete proteger en forma segura y anónima las denuncias para ulterior investigación y eventual exposición al escrutinio público. Es una protección contra represalias de parte de los grupos afectados, quienes pueden reaccionar severamente ante aquellos que han develado su proceder clandestino. En lo concreto, dicha plataforma ofrece el recurso denominado Chileleaks para una denuncia segura, la cual es un buzón online seguro y anónimo el cual, utilizando el browser TOR, recibe alertas de cohecho (sobornos) protegiendo la identidad del denunciante ${ }^{5}$, quien debe aportar pruebas suficien-

\footnotetext{
${ }^{5}$ Protege las direcciones IP, si y solo si, se evita el uso de
}

tes para impulsar una investigación subsecuente por parte de un medio de comunicación.

Otra iniciativa semejante es ALAC, (Advocacy and Legal Advice Centre), una red de oficinas que desde 2003 en el mundo, han atendido a más de 200.000 personas hasta la fecha de esta edición. Actualmente hay más de 100 oficinas ALAC en 60 países con la misión de impulsar la participación ciudadana. Actúa como facilitador entre el ciudadanola ciudadanía y las autoridades locales para atender quejas sobre corrupción, además de consolidar reformas legales e institucionales para fortalecer la integridad pública y la capacidad institucional para detectar y sancionar actos de corrupción. En Chile, ALAC, quien es apoyada por el Ministerio Público, la Universidad de Chile y la Contraloría General de la República, ha recibido denuncias a través de la plataforma www.denunciacorrupción.cl y vía WhatsApp (54 denuncias en un año), siguiendo el procedimiento de: orientación, identificación de autoridad competente para focalizar y encauzar eficientemente la denuncia, seguimiento del caso y garantía de confidencialidad de la información entregada.

Así mismo en distintas partes del mundo han emergido iniciativas de control social que, en la forma de entidades como Ciudadano Inteligente, Observatorios Sociales (en Chile, Colombia, Brasil), han evitado que millones de dólares caigan en manos equivocadas. A estas habría que sumar iniciativas como The Global Compact de ONU, con su principio N 10 "Lucha contra la Corrupción"; BID, con su apartado de Transparencia de Denuncias de fraude y corrupción; OCDE, con su Programa Anticorrupción; y las recomendaciones del Banco Mundial en torno a la transparencia con datos abiertos y uso de las últimas tecnologías para luchar contra la corrupción, como lo es el blockchain. A través de esta tecnología, señalada como la cuarta revolución industrial, es una "cadena de bloques digitales que contiene información" (Banco Mundial, 2019); la que, una vez generada, es muy complejo modificarla ya que sus operaciones (datos del remitente, destinatario y monto de dinero), están gobernados por un estricto marco de normas y claves criptográficas.

Nada de lo aquí expuesto es de complejidad inconmensurable que frene las ansias para enfrentar el comportamiento corrupto en las organizaciones, de modo que, tampoco, nada obsta para impulsarlas.

software como Flash, RealPlayer, Quicktime y otros. TOR está disponible para usarse desde aplicativos Android e iOS 


\section{Referencias}

Association of Certified Fraud Examiners (2018). Report to the nations. Global study on occupational fraud and abuse.

Banco Mundial (2019). Blockchain: cómo asegurarse que cada dólar llegue a quien lo necesita. Recuperado en: https://www.bancomundial.org/.

Beltrán, J., Carmona, M., Carrasco, R., Rivas, M., y Tejedor, F. (2002). Guía para una gestión basada en procesos. Instituto Andaluz de Tecnología.

Boniolo, P. (2010). The thread of corruption: a study of the middle and working classes in buenos aires. Revista Mexicana de Sociología, 72(3):365391.

CEPAL y Ministerio de Desarrollo Social de Chile (2017). Compendio del Primer Congreso Internacional de Evaluación Social de Proyectos.

Goncalvez Panta, D. (2016). Servicio de ingeniería inversa para el desarrollo de proyectos qalia. Tesis de máster, Facultad de Economía y Negocios, Universidad de Chile.

Heidenheimer, A. J., Johnston, M., y Le Vine, V. T. (1989). Political corruption: A handbook. Transaction Publisher, New Brunswick.

Kepner, C. H. y Tregoe, B. B. (1965). El Directivo Racional. Mc Graw-Hill.

KPMG International (2016). Global profiles of the fraudster, Technology enables and weak controls fuel the fraud.

Martínez Rosón, M. d. P. (2016). I prefer the corrupt one: A profile of citizens who choose dishonest but competent politicians. Revista Española de Investigaciones Sociológicas.

Mora, J., Huilcapi, M., Gil, D., Camacho, W., y Uhsca, N. (2017). Corruption versus forensic audit, to economic development in a social system without moral precepts. Journal of Science and Research:Revista Ciencia e Investigación, $2(7): 37-45$.

Mujica, J. (2004). Palabras corruptas: El lenguaje de la corrupción o la corrupción como lenguaje. Anthropia, (3).

Mujica, J. (2005). Relaciones corruptas: Poder, Autoridad y Corrupción en Gobiernos Locales.
Navea, L. (2014). Fundador de transparencia internacional, peter eigen: "'chile puede ser un modelo anticorrupción en el sector minero". $L a$ Segunda online.

Pardinas, F. (1984). Metodología y técnicas de investigación en ciencias sociales. Siglo veintiuno editores.

PriceWaterhouseCoopers (2010). Fraude en el sector público. Centro $\mathrm{PwC}$ de Investigación del Sector Público.

Transparencia Internacional (2009). El Informe Global de la Corrupción 2009. Transparencia Internacional.

Villoria, M. (2000). Ética pública y corrupción: Curso de ética administrativa. Editorial Tecnos. 